\title{
Promoción De La Salud En Personas Sordas Mayores De La Comunidad Sorda
}

\author{
Promoção Da Saúde Em Pessoas Idosas Surdas Na \\ Comunidade Surda
}

\section{Health Promotion In Elderly Deaf People Of The Deaf Community}

iD Meritxell Segovia-Quiñones Universitat de Barcelona

iD

Vera Ponce-López Universitat de Barcelona

Neuma Chaveiro

Universidade Federal de Goiás

(iD)

Eva Gómez-Rodríguez

Universitat de Barcelona

Dolors Rodríguez-Martín

Universitat de Barcelona

Resumo: Este artigo apresenta uma experiência na área da saúde mostrando um projeto de promoção da saúde em colaboração com a Comunidade Surda e dirigido a idosos surdos. O projeto foi realizado por estudantes de enfermagem em seu Projeto de Graduação na modalidade Aprendizagem e Serviço. Uma intervenção enquadrada em um programa de educação em saúde que consistia em 3 sessões de $2 \mathrm{~h}$ foi realizada com um grupo de 20 idosos surdos em que aspectos do envelhecimento saudável (atividade e exercícios físicos; 
alimentação saudável) foram abordados de forma teórica e prática ; sono e repouso saudáveis; e patologias prevalentes na população). A experiência revelou-se muito satisfatória tanto para os alunos como para a Comunidade Surda, resultando nesta experiência muito enriquecedora tanto ao nível da aprendizagem e aquisição de competências no trabalho comunitário, como ao nível do contacto com a Comunidade Surda.

Palavras chave: Aprendizagem-serviço. Treinamento de enfermagem. Promoção da saúde. Surdos. Idosos.

Abstract: This paper exposes an experience in the field of health showing a health promotion project in collaboration with the Deaf Community and addressed to elderly deaf people. Nursing students carried out this project in their Final Degree Project in the Service-Learning modality. An intervention was framed in a health education program, that consisted of 3 sessions of 2 hours each, it was carried out with a group of 20 elderly deaf people in which aspects of healthy aging were addressed in a theoretical and practical way (physical activity and exercise; healthy eating; healthy sleep and rest; and diseases prevalent in the population). For the students and for the Deaf Community the experience turned out to be very satisfactory, resulting in this very enriching experience at the level of learning and acquisition of skills in community work, as well as at the level of contact with the Deaf Community.

Key words: Service-Learning. Nurse training. Health Promotion. Deaf. Ederly People.

Resumen: El presente artículo presenta una experiencia en el ámbito de la salud mostrando un proyecto de promoción de la salud en colaboración con la Comunidad Sorda y dirigido a personas mayores sordas. El proyecto fue llevado a cabo por alumnas de Enfermería en 
su Trabajo de Fin de Grado en la modalidad de Aprendizaje y Servicio. Se realizó una intervención enmarcada en un programa de educación para la salud que constó de 3 sesiones de 2 h con un grupo de 20 personas mayores sordas en los que se abordaron de forma teórica y práctica aspectos de envejecimiento saludable (actividad y ejercicio físico; alimentación saludable; sueño y descanso saludables; y patologías prevalentes en la población). La experiencia resultó ser muy satisfactoria tanto para las alumnas como para la Comunidad Sorda, resultando esta experiencia muy enriquecedora tanto a nivel de aprendizaje y adquisición de competencias en el trabajo comunitario, como a nivel de contacto con la Comunidad Sorda.

Palabras clave: Aprendizaje-servicio. Formación enfermera. Promoción de la salud. Sordos. Ancianos.

Data de submissão: 23/11/2020

Data de aprovação: 09/12/2020 
Promoción de la salud en personas sordas mayores de la comunidad sorda Meritxell Segovia-Quiñones • Vera Ponce-López, et al...

\section{Introducción}

En el presente artículo presentamos la experiencia de una intervención de promoción para la salud centrada en el colectivo de personas mayores de la Comunidad Sorda llevado a cabo en Barcelona (España). Esta intervención se realizó en el contexto del Trabajo de Final de Grado de Enfermería de la Universitat de Barcelona (UB) y fue proyectado, ejecutado y evaluado por dos alumnas de $4^{\circ}$ curso del Grado de Enfermería, bajo la supervisión y tutorización de 3 profesoras de la UB siendo una de ellas investigadora postdoctoral en la UB y profesora de la Univesidade Federal de Goiás (UFG).

La intervención llevada a cabo, se realizó gracias a la propuesta pedagógica en la Universitat de Barcelona, como es el Aprendizage y Servicio (ApS) que se lleva a cabo en la UB. El ApS es una propuesta educativa y de investigación que incluye tanto el servicio comunitario, como el aprendizaje académico, en lo que sería un único proyecto en el que el alumnado se forma a medida que trabaja para satisfacer las necesidades reales del entorno con el objetivo de mejorarlo (PUIG et al., 2009).

Tanto el diseño como la implementación de los proyectos de ApS, requieren de una estrecha vinculación entre profesorado, alumnado, entidades colaboradoras y ciudadanía. Por este motivo, el punto de partida para poder llevarlos a cabo es la estrecha colaboración entre estos agentes, para que conjuntamente sea posible responder a las necesidades del entorno, aprovechando las potencialidades de cada persona participante. (MORIN et al., 2017a)

En esta experiencia que presentamos, la entidad que colabora es la Federació de Persones Sordes de Catalunya (FESOCA), junto con la que se realizarán actividades orientadas a favorecer cuidados enfermeros a las personas mayores miembros de la Co- 
Promoción de la salud en personas sordas mayores de la comunidad sorda Meritxell Segovia-Quiñones • Vera Ponce-López, et al...

munidad Sorda catalana ya que uno de los propósitos de la formación de los estudiantes de enfermería es capacitarlos para cuidar y promover la salud comunitaria, entendida como todas las estrategias, métodos y actividades encaminadas a mejorar la salud de la población (MORIN et al., 2017b).

Así, en este trabajo se enfocó plantear una serie de intervenciones para la promoción de la salud de éste colectivo, centrándonos en las demandas del mismo y tratando de proporcionar el máximo de recursos y herramientas posibles para que fueran las propias personas las responsables directas de su salud y, por tanto, conocedoras de todo cuanto se refiere a esta.

\section{Marco Teórico}

\section{Sordera desde la perspectiva biomédica y la perspecti- va sociolingüística-cultural}

Según la Organización Mundial de la Salud (OMS) se califica de pérdida de audición la incapacidad de oír "tan bien como una persona cuyo sentido del oído es normal, es decir, cuyo umbral de audición en ambos oídos es igual o superior a 25 dB" (OMS, 2019a).

La sordera puede clasificarse según el grado de pérdida auditiva, según su etiología, en función de si la pérdida se dio antes o después de la adquisición de la lengua oral, etc. Además también hay que conocer que hay personas con sordera que utilizan audífonos, implantes cocleares u otros dispositivos para mejorar la comunicación, que se pueden comunicar tanto en lenguas orales como en lenguas de signos o bien ambas.

A nivel mundial, la OMS estima que aproximadamente más del $5 \%$ de la población mundial (466 millones de personas) padece pérdida de audición discapacitante (432 millones de adultos y 34 
Promoción de la salud en personas sordas mayores de la comunidad sorda Meritxell Segovia-Quiñones • Vera Ponce-López, et al...

millones de niños). Entendiendo la pérdida de audición discapacitante como: "aquella superior a $40 \mathrm{~dB}$ en el oído con mejor audición en los adultos, y superior a 30 dB en el oído con mejor audición en los niños" (OMS, 2019a). Además, la propia OMS estima que una tercera parte de éstas personas son mayores de 65 años. La World Federation of Deaf (WFD) estima que alrededor de 70 millones de personas sordas en el mundo son usuarias de las diferentes lenguas de signos ya sea como primera lengua o como lengua materna. La lengua de signos no es una lengua universal, sino que existen 144 catalogadas a nivel mundial. Por poner unos ejemplos, en EEUU se estima que hay alrededor de 250.000 personas usuarias de la American Sign Language (ASL); en Brasil entre 200.000 y 1.000.000 de personas son usuarias de la Língua Brasileira de Sinais (LIBRAS); en el Estado español, entre 45.000 y 75.000 personas usuarias de la Lengua de Signos Española (LSE); y entre 9.00015.000 usuarias de la Llengua de Signes Catalana (LSC) en Cataluña (CDC, 2019; EBERHARD et al., 2019; OMS, 2019b; WFD, 2019).

Al concepto de sordera nos podemos aproximar desde dos posicionamientos antagónicos, como son la perspectiva biomédica y la perspectiva sociolingüística-cultural.

La perspectiva biomédica, entiende la sordera como un déficit, que deviene en una discapacidad, por lo tanto una alteración de lo que se considera como normal. Así, la sordera, se considera como una alteración patológica y precisa de intervención médica para poder ser diagnosticada y tratada. Asimismo, la sordera no es considerada como un problema únicamente por el déficit auditivo, sino también por lo que implica y repercute en la comunicación con otras personas, por este motivo otro de los objetivos de esta perspectiva es que la persona adquiera el habla oral. Desde este enfoque las personas sordas han sido tratadas como sujetos pasivos sin tener en cuenta sus opiniones o bien necesidades (GUTIERREZ et al., 2016; RODRIGUEZ-MARTíN, 2013). 
Promoción de la salud en personas sordas mayores de la comunidad sorda Meritxell Segovia-Quiñones • Vera Ponce-López, et al...

La aproximación desde la perspectiva sociolingüística-cultural -que es la propia que surge de las personas con sordera usuarias de las lenguas de signos- entenderá a las personas sordas como un grupo social y cultural minoritario con una identidad cultural propia, que surge como consecuencia de participar de un conjunto de experiencias como personas con sordera y que además comparten una lengua común que es la lengua de signos. Es decir, una comunidad, con su lengua, historia y cultura: la Comunidad Sorda. Esta comunidad percibe la realidad principalmente a través del rasgo visual, el cual constituye el eje central de su comunicación, la lengua de signos, como lengua gesto-visual que aparece de forma natural como aspecto de adaptación sensorial (RODRIGUEZ-MARTíN, 2013).

La Comunidad Sorda es heterogénea, incluye a individuos de cualquier condición personal o social. Los dos elementos principales que definen esta comunidad son la lengua de signos y la identidad que dispone. En primer lugar, la lengua de signos es un elemento de cohesión del grupo y que cubre las necesidades comunicativas y de convivencia de sus miembros. La lengua de signos incluye tanto el alfabeto manual, la postura y expresión corporal, el movimiento de las manos y las expresiones faciales. Por lo tanto, al contrario de lo que creen muchas personas, es una lengua natural y viva, que nace del encuentro entre las personas sordas, y por tanto, posee las mismas características lingüísticas, gramaticales y sintácticas que cualquier otra lengua, con la particularidad de que es una lengua espacial. En segundo lugar, el otro elemento característico de esta comunidad es su sentimiento identitario como fruto de su historia común y de la interacción entre sus miembros (CHAVEIRO et al. 2020; RODRIGUEZ-MARTíN, 2013). 
Promoción de la salud en personas sordas mayores de la comunidad sorda Meritxell Segovia-Quiñones • Vera Ponce-López, et al...

\section{Promoción de la salud}

Esta experiencia que presentamos en este artículo se encuentra enmarcada dentro de un proyecto de promoción de la salud. La OMS define la promoción de la salud como "el proceso que confiere a la población los medios para incrementar el control de su propia salud con tal de poderla mejorar". Por lo tanto, no solo dirige acciones centradas en el tratamiento y la curación, sino que también abarca intervenciones sociales, ambientales y económicas de los diferentes sectores, destinadas a mejorar el entorno global, la calidad de vida de las personas y a prevenir e incidir sobre los factores causantes de los problemas de salud (OMS 2016).

La promoción de la salud, por tanto, ayuda a fomentar estilos y hábitos de vida saludable, ya que permite concienciar a la población sobre determinados comportamientos que podrían ser perjudiciales tanto para su salud como para la del entorno.

En este proyecto se llevo a cabo un programa de Educación para la Salud como herramienta de la promoción de la Salud, ya que la educación para la salud es un recurso que que combina la responsabilidad individual, colectiva y social en el mantenimiento de conductas y condiciones de vida que promueven la salud y la calidad de vida de las personas.

\section{Envejecimiento saludable}

Envejecimiento activo es un término utilizado por la OMS que se define por el proceso de optimizar las oportunidades de salud, participación y seguridad con la finalidad de mejorar la calidad de vida de las personas que envejecen (AZUA, 2011; CABEZAS-PEÑA et al 2014; OMS 2019c).

Por definición el envejecimiento consiste en la acumulación de daños moleculares y celulares a lo largo del tiempo y de los años. Este fenómeno tiene como consecuencia la disminución de 
Promoción de la salud en personas sordas mayores de la comunidad sorda Meritxell Segovia-Quiñones • Vera Ponce-López, et al...

las capacidades físicas y mentales, un aumento del riesgo de enfermedad y la muerte (ANGARITA et al. 2017; OMS, 2019c).

Según la OMS el entorno y los hábitos de vida son dos factores que influyen mucho en el envejecimiento saludable. En primer lugar, el entorno en el que vives facilita o dificulta la adquisición de hábitos de vida saludables dependiendo si en el entorno están inculcados, si hay recursos disponibles o accesibilidad a servicios. Por otro lado, estos hábitos de vida saludables también influyen ya que está demostrado que si se realizan desde una edad temprana el envejecimiento será más saludable y favorecedor (ANGARITA et al. 2017; CABEZAS-PEÑA et al 2014).

\section{Personas sordas mayores en el ámbito de la salud}

Los sistemas de salud, así como los servicios contenidos en él se encuentran poco adaptados a la diversidad, por tanto, es importante tener en cuenta determinados aspectos para poder atender las necesidades y demandas de las personas con sordera. Es importante tener presente que no todas las personas con sordera tienen las mismas necesidades y que no todas son usuarias de las lenguas de signos, por lo tanto los servicios y profesionales de la salud han de saber que lengua utilizan para expresarse y comunicarse, que características individuales presentan, etc. Por otro lado, aun siendo todas ellas distintas, cualquiera de ellas independientemente de sus situaciones o necesidades, requieren de un fácil acceso a la información de nuestro entorno sin ningún tipo de barreras o impedimentos (CHAVEIRO et al. 2010; MUÑOZ-BAELL et al. 2011; RODRIGUEZ-MARTíN et al. 2018).

En lo que respecta a las personas mayores sordas usuarias de las lenguas de signos, no existen datos estadísticos ni epidemiológicos referidos este sector de la población. Si nos referimos a términos generales, la OMS estima que actualmente hay casi 700 
Promoción de la salud en personas sordas mayores de la comunidad sorda Meritxell Segovia-Quiñones • Vera Ponce-López, et al...

millones de personas en el mundo que son mayores de 60 años, calculando que para 2050 hasta más de 2000 millones de personas estarán en esta franja de edad (OMS 2019c).

Estos datos remarcan el proceso de envejecimiento que está teniendo la población a nivel mundial en la actualidad. Este envejecimiento, además, también es una muestra de mejora de salud en el mundo, y en consecuencia el aumento de la esperanza de vida en la población. Pero, por otro lado, hay que adaptar los servicios y la atención social prestada, a las necesidades específicas de las personas mayores.

Paulino Azúa explica

Buena parte de las personas mayores se enfrentan a un doble reto: el propio proceso de envejecimiento y la indiferencia (...) Pero las personas mayores con discapacidad tienen ante sí un tercer obstáculo: la propia discapacidad que, por lo general, les sitúa en desigualdad de oportunidades. (AZUA, 2012, p.12).

Por consiguiente, el entorno de las personas mayores sordas usuarias de las lenguas de signos suele estar afectado por las barreras de comunicación ya existentes y un deficitario acceso a la información. Pero, además, las personas sordas tienen una serie de dificultades añadidas a su día a día. Cada día se encuentran con barreras comunicativas o discriminatorias en la sociedad oyente mayoritaria, pero además, si hablamos de personas mayores, que pueden tener asociadas enfermedades crónicas, la complejidad es aún mayor (AZUA, 2011).

Las personas mayores suelen tener dificultades de desarrollo de la autonomía personal y dependencia, si además son sordas, la participación y formación suelen ser deficitarias debido a las barreras con las que se encuentran. El envejecimiento debe ir conec- 
Promoción de la salud en personas sordas mayores de la comunidad sorda Meritxell Segovia-Quiñones • Vera Ponce-López, et al...

tado a la prevención de situaciones de dependencia mediante la actividad física diaria, la alimentación saludable y en general a los estilos de vida saludables. Se debe fomentar el desarrollo de todo su potencial y gestionar el tiempo libre del que disponen de forma saludable y haciendo actividades de ocio y recreativas (ANGARITA et al. 2017).

Con las personas mayores sordas, también encontramos que suelen tener más dificultades si tienen alguna enfermedad crónica ya que tanto la medicación como la información sobre la propia enfermedad no suele darse de forma accesible (AZUA, 2011).

Por todo ello es importante, crear planes y actividades, dentro de lo que sería la educación para la salud, que estén adaptados a esta comunidad, para así fomentar el empoderamiento en cuanto a la participación y autonomía en el propio autocuidado.

\section{Justificación del proyecto}

A partir de todo lo desarrollado en el apartado del marco teórico se justifica el desarrollo este trabajo, sobre todo teniendo en cuenta que las alumnas como futuras enfermeras toman conciencia de las dificultades que pueden encontrarse las personas mayores sordas a nivel sanitario, para de este modo, tratar de revertirlas o disminuir posibles consecuencias en su salud. Para ello se parte de que enfermería tiene un papel fundamental en la promoción de la salud, pero en el caso de personas no oyentes esta función se complica y se multiplican las barreras sociolingüísticas y comunicativas ya existentes. Este colectivo, es consciente de estas dificultades y lucha por sus derechos para poder tener una mayor calidad de vida. Pero, aun así, se pueden dar casos de personas sordas que no tienen tantos recursos a la hora de comunicarse, por baja competencia lecto-escritora, la falta de disponibilidad de los servicios de interpretación, etc. 
Promoción de la salud en personas sordas mayores de la comunidad sorda Meritxell Segovia-Quiñones • Vera Ponce-López, et al...

Así pues, este trabajo surge a raíz de la demanda que hacen las mismas personas sordas mayores a través de la FESOCA, para aumentar sus conocimientos sobre hábitos de vida saludable, prevención y detección de enfermedades o determinados factores de riesgo para su salud.

Por tanto, lo que se pretende es solucionar cualquier duda o temor que puedan presentar estas personas en relación a si llevan unos hábitos de vida saludables, facilitarles información a la que probablemente no han tenido un fácil acceso y evitar conductas de riesgo para su estado de salud y su bienestar tanto físico como mental.

\section{Metodologia}

\section{Necesidades y datos de la comunidad}

Para determinar las necesidades y demandas de las perso12 nas mayores que forman parte de la Comunidad Sorda en Catalunya (España), se realizó una reunión con la responsable de la Vocalía personas mayores de la Federación de Persones Sordes de Catalunya (FESOCA). En esta reunión se determinaron las necesidades en cuanto a temas de salud - de las personas mayores sordas- para poder elaborar un programa de educación para la salud con el objetivo de enfocar las intervenciones que realizaríamos. Después de la reunión se decidieron conjuntamente los temas que se trabajarían en las intervenciones. Finalmente se acordó que las intervenciones serían 3 sesiones de $2 \mathrm{~h}$ de duración sobre aspectos referidos al envejecimiento saludable en los que se trabajarían los temas de: el ejercicio y actividad física; la alimentación; y patologías prevalentes en los y las participantes de las sesiones. Las intervenciones fueron diseñadas y planificadas para que fueran totalmente accesibles, teniendo en cuenta la aparición de posibles dificultades de comunicación y comprensión de la información proporcionada. 
Promoción de la salud en personas sordas mayores de la comunidad sorda Meritxell Segovia-Quiñones • Vera Ponce-López, et al...

En relación a la búsqueda de información sobre la salud de las personas sordas en la etapa de la vejez, referido a sus estilos de vida y a los recursos sanitarios que más utilizan, no se encontraron muchos resultados o fuentes fiables al respecto. Esto puede indicar lo poco que se conoce este tema y la escasa investigación que se ha llevado en el ámbito de la salud sobre personas mayores de la Comunidad Sorda. Por este motivo, cabe destacar la importancia de realizar estas intervenciones y tratar de educar a la población sorda sobre los aspectos más relevantes a tener en cuenta para su salud en este periodo del ciclo vital.

Ante la falta de información y datos concretos sobre salud y personas sordas mayores, nos centramos en los datos con respecto a la población de personas mayores a nivel general. Así, estos datos también nos fueron de gran ayuda para enfocar las sesiones con personas mayores sordas.

Las causas más frecuentes por la que las personas mayores precisan asistencia sanitaria, de mayor a menor prevalencia son las cardiovasculares, respiratorias, digestivas y las neoplasias, pero de entre éstas, las que causan más mortalidad entre la población mayor son en primer lugar las cardiovasculares, en segundo lugar, los tumores y en tercer lugar las respiratorias, a pesar de que en los últimos años ha aumentado la mortalidad por enfermedades de salud mental. A pesar del aumento de esperanza de vida en edades avanzadas, en España el 85,6\% de los fallecidos son personas mayores (DEPARTAMENT DE SALUT, 2019).

\section{Objetivos generales y específicos del servicio Objetivo general:}

Fomentar el envejecimiento saludable en la comunidad sorda mediante un programa de educación para la salud. 
Promoción de la salud en personas sordas mayores de la comunidad sorda Meritxell Segovia-Quiñones • Vera Ponce-López, et al...

\section{Objetivos secundarios:}

1. Incrementar los conocimientos sobre actividad y ejercicio físico en las personas mayores de la Comunidad Sorda catalana.

2. Incrementar la actividad física y el ejercicio físico en las personas mayores de la Comunidad Sorda catalana.

3. Incrementar los conocimientos sobre alimentación saludable adaptada a las personas mayores de la Comunidad Sorda catalana.

4. Fomentar que las personas mayores de la Comunidad Sorda catalana sea autonoma en la creación de menús de saludables.

\section{Planificación y realización del servicio}

Se planificaron y llevaron a cabo tres intervenciones dirigidas a un grupo de personas sordas de la tercera edad, usuarias de la Llengua de Signes Catalana (LSC). Las 3 sesiones tenían una duración de 2 horas y se realizaron en un aula de la Universitat de Barcelona en marzo de 2019.

El reclutamiento de los/as participantes se realizó a través de la FESOCA. La responsable de la vocalía de personas mayores grabó un video de difusión en LSC para invitar y animar a las personas sordas a participar en las sesiones. Finalmente asistieron un total de 20 participantes de entre 60 y 80 años, siendo el $67 \%$ mujeres y el $33 \%$ hombres.

Las sesiones se realizaron de forma dinámica y mediante el uso de recursos adecuados a la población a quien iban dirigidas - personas mayores de la Comunidad Sorda_, con tal de que al finalizar las sesiones cada uno de los/as participantes hubiera adquirido conocimientos y habilidades de utilidad para poder aplicarse a su vida diaria. 
Promoción de la salud en personas sordas mayores de la comunidad sorda Meritxell Segovia-Quiñones • Vera Ponce-López, et al...

\section{Intervenciones}

Las sesiones se realizaron de forma dinámica y mediante el uso de recursos adecuados a la población a quien iban dirigidas - personas mayores de la Comunidad Sorda-, con tal de que al finalizar las sesiones cada uno de los/as participantes hubiera adquirido conocimientos y habilidades de utilidad para poder aplicarse a su vida diaria.

La estructura de las sesiones era común a todas ellas, en la primera parte de las mismas se realizaba la parte teórica y demostrativa a través de ejemplos prácticos. Después se abría un espacio para el diálogo, aportación de experiencias propias y posibles dudas. En la parte final de cada sesión se realizaba una valoración de lo aprendido y de las posibles preocupaciones o dificultades que pudieran aparecer. De esta manera se podía observar si la información dada se había entendido correctamente y si les había servido de ayuda para mejorar su estado de salud.

Además, al final de cada sesión y con el fin de promover una alimentación saludable, de interactuar con el grupo y de crear una relación más cercana con cada uno de ellos/as, al finalizar cada intervención se hace un pequeño piscolabis con varios tipos de fruta de temporada y frutos secos.

\section{Sesión 1: Actividad y ejercicio físico.}

En el inicio de esta sesión se realizó la presentación de la intervención de promoción para la salud centrada en el envejecimiento saludable, de cómo se iban a organizar las sesiones y a continuación se dio un pequeño espacio para que los/as participantes se presentaran y explicaran si padecían algún problema de salud. Además de ello, se pasó una encuesta inicial, con el fin de conocer los intereses y particularidades de los/as participantes, así 
Promoción de la salud en personas sordas mayores de la comunidad sorda Meritxell Segovia-Quiñones • Vera Ponce-López, et al...

como temas de salud que les interesaban, para poder trabajarlos en las sesiones posteriores. De entre los temas surgidos de esta encuesta inicial, se consensuó que durante la $2^{\mathrm{a}}$ sesión —en que se trabajaría el tema de alimentación - también se abordaría el tema del colesterol, el ritmo deposicional y el estreñimiento. Para la última sesión, se consensuó que se trabajaría sobre el descanso y sueño saludables, así como el insomnio. Pero, además también hicieron la demanda, en esta encuesta inicial, de que se hablara sobre los signos y síntomas de patologías como son el infarto agudo de miocardio y los accidentes cerebrovasculares y las actuaciones o bien procedimientos que se llevan a cabo en estos casos.

Los resultados de la encuesta pasada en esta primera sesión mostraron que un $25 \%$ que se habían inscrito por curiosidad y cerca de un 50\% por aprender. Cuando fueron preguntados sobre sus patologías crónicas un 28\% padecía de Hipertensión Arterial, un $11 \%$ Diabetes Mellitus; y el 5\% Cáncer. Al ser preguntados sobre si pensaban que estas sesiones les ayudarían a estar sanos, el $87 \%$ opinó que sí y un 13\% dejó la respuesta sin responder.

Después de pasar la encuesta se trabajó sobre la actividad y el ejercicio físico adaptado a las necesidades de las personas mayores, aportando aspectos teóricos y prácticos. Se realizó una parte práctica de ejercicios de calentamiento y estiramientos para que pudieran adaptar y realizar en sus domicilios como rutinas diarias. Estos ejercicios se adaptaron a los problemas osteomusculares que algunos/as de las participantes padecían y se mencionaron aspectos a tener en cuenta al realizarlos, como por ejemplo: tipo de ropa para realizarlos, estar atentos a la aparición de dolor y que hacer ante ello, etc. 
Promoción de la salud en personas sordas mayores de la comunidad sorda Meritxell Segovia-Quiñones • Vera Ponce-López, et al...

\begin{tabular}{|l|l|}
\hline Contenidos trabajados & Objetivos \\
\hline $\begin{array}{l}\text { Diferenciar entre actividad y } \\
\text { ejercicio físico. } \\
\text { Beneficios actividad física en el } \\
\text { envejecimiento. }\end{array}$ & $\begin{array}{l}\text { Los participantes de la sesión serán } \\
\text { capaces de diferenciar los concep- } \\
\text { tos entre actividad física y ejercicio } \\
\text { físico. } \\
\text { des de la vida diaria. } \\
\begin{array}{l}\text { Práctica de ejercicios dinámi- } \\
\text { cos de calentamiento y estira- } \\
\text { mientos. }\end{array}\end{array}$ \\
$\begin{array}{l}\text { Compartirán los hábitos de actividad } \\
\text { física que suelen hacer cada día para } \\
\text { poder avaluar si son correctos o no y } \\
\text { valorar si habría que aumentar esta } \\
\text { actividad. } \\
\text { Durante la realización de la parte } \\
\text { práctica se imitarán los movimientos } \\
\text { demostrativos. } \\
\text { Demostrarán que han entendido el } \\
\text { contenido en la síntesis final de la } \\
\text { sesión. }\end{array}$ \\
\hline
\end{tabular}

Tabla 1: Contenidos y objetivos primera sesión.

Fuente: Elaborado por las autoras

\section{Sesión 2: Alimentación saludable en personas mayores}

Gracias a la primera sesión y a la realización de la encuesta inicial, se pudo observar el gran interés que presentaban todos/as los/as participantes por conocer lo que es y lo que implica llevar una alimentación saludable. La sesión se inició con la parte más teórica sobre aspectos de alimentación saludable, grupos de alimentos y adaptación a los grupos de edad.

Algunos/as participantes expusieron sus casos concretos e hicieron peticiones sobre temas como el colesterol, patrones deposicionales normales y alterados, la tos crónica etc. De esta manera, la sesión se centró también en estas demandas de los/as participantes, teniendo en cuenta el rango de edad de éstos, a la hora de relacionar los grupos de alimentos y sus propiedades con los cambios y alteraciones que aparecen en la vejez.

Los/as participantes mostraron un gran interés por todo el contenido de la sesión, fueron realizando preguntas y compartien- 
Promoción de la salud en personas sordas mayores de la comunidad sorda Meritxell Segovia-Quiñones • Vera Ponce-López, et al...

do vivencias sobre lo que ellos hacían en sus casas o trucos que a ellos les iba bien. Fue una sesión muy dinámica y entretenida a pesar de su elevado contenido teórico, especialmente gracias a las dudas y la participación activa del grupo.

Una de las recomendaciones por las que mostraron gran curiosidad fue la del plato saludable, ya que la mayoría coincidía en que suelen realizar platos sencillos y poco elaborados para una mayor comodidad y rapidez, pero de forma saludable.

Otro aspecto sobre el cual presentaron dudas era el calcio y los tipos de leche, ya que a muchos participantes no les sentaba bien la leche de vaca, por lo que comentaron que la sustituyen por bebidas vegetales como la soja, avena, etc.

18

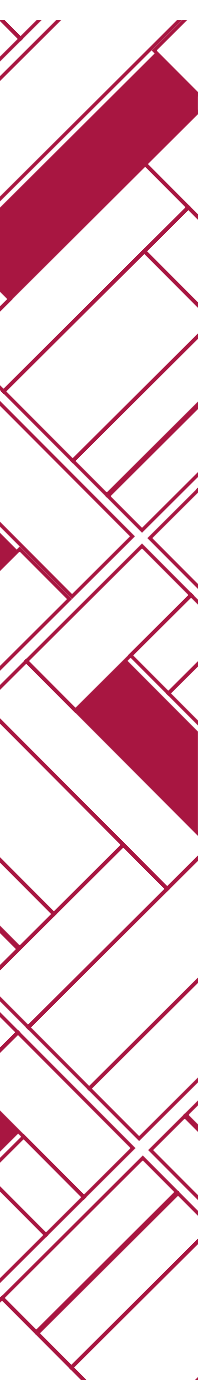

Objetivos

- Los participantes sabrán reconocer los alimentos adecuados y más importantes para mantener una alimentación saludable.

- Detectaran y reconocerán si sus hábitos son los adecuados y como cambiarlos en caso de que no lo sean.

- Sabrán poner ejemplos de alimentación sana.

- Demostrarán que han entendido el contenido en la síntesis final de la sesión.

Tabla 2: Contenidos y objetivos tercera sesión.

Fuente: Elaborado por las autoras.

\section{Sesión 3. Sueño/descanso e insomnio en la tercera edad y otros problemas de salud.}

En esta sesión en que se trabajó el sueño/descanso e insomnio, muchos participantes manifestaron sus dificultades a la hora de conciliar el sueño o la duración del mismo. Además, cuando 
Promoción de la salud en personas sordas mayores de la comunidad sorda Meritxell Segovia-Quiñones • Vera Ponce-López, et al...

se explicaron las diferentes recomendaciones según los estudios consultados, para mejorar o facilitar el sueño, algunos de los/as participantes también compartieron sus trucos para conciliar el sueño. Otros, debatían las diferentes rutinas que tenían antes de ir a dormir y si estas eran beneficiosas o no.

Por otro lado compartían las diferentes rutinas que tenían antes de ir a dormir y esto nos facilitó poder modificar las conductas inapropiadas o que dificultan el sueño como por ejemplo ver la televisión o utilizar los dispositivos móviles antes de ir a dormir ya esta rutina dificulta la conciliación del sueño a posteriori. Ante estos hábitos identificados se proponían alternativas o realizar técnicas de relajación antes de ir a dormir con el objetivo de facilitar el descanso.

En la parte final de la sesión se trabajó sobre los temas propuestos por los/as participantes como fueron los infartos agudos de miocardio y los accidentes cerebro vasculares. El grupo tenía mucho interés en sus manifestaciones clínicas para poder detectar estos síntomas en su propia persona o bien en sus cónyuges/parejas. Además insistieron mucho en saber cómo actuar ante estas manifestaciones clínicas con el objetivo de minimizar la gravedad y acudir lo antes posible a un centro sanitario.

\begin{tabular}{|c|c|}
\hline Contenidos trabajados & Objetivos \\
\hline $\begin{array}{l}\text { - Describir los beneficios del sueño } \\
\text { y descanso saludable. } \\
\text { Explicar los síntomas del insom- } \\
\text { nio y concepto del mismo. } \\
\text { Explicar las situaciones que } \\
\text { dificultan un correcto descanso } \\
\text { nocturno. } \\
\text { Recomendaciones que pueden } \\
\text { ayudar al sueño nocturno. } \\
\text { Práctica de relajación, para que } \\
\text { la puedan realizar en sus casas. } \\
\text { Explicación sobre los infartos } \\
\text { agudos de miocardio y los acci- } \\
\text { dentes cerebrovasculares.. }\end{array}$ & $\begin{array}{l}\text { - Los participantes serán capaces de } \\
\text { describir los beneficios de un buen } \\
\text { descanso nocturno mediante la puesta } \\
\text { en común. } \\
\text { - Compartirán verbalmente sus cono- } \\
\text { cimientos o hábitos antes de irse a } \\
\text { dormir para verificar entre todos si es } \\
\text { un hábito positivo o negativo para el } \\
\text { descanso. } \\
\text { - Los participantes de la sesión serán } \\
\text { capaces de realizar la técnica de relaja- } \\
\text { ción de forma autónoma. } \\
\text { Demostrarán que han entendido el } \\
\text { contenido de la sesión mediante la } \\
\text { puesta en común. }\end{array}$ \\
\hline
\end{tabular}

Tabla 3: Contenidos y objetivos tercera sesión.

Fuente: Elaborado por las autoras.

Revista UFG. 2020, v.20: e20.66751 
Promoción de la salud en personas sordas mayores de la comunidad sorda Meritxell Segovia-Quiñones • Vera Ponce-López, et al...

\section{Evaluación del programa de educación para la salud}

La evaluación de las sesiones se realizó mediante la observación, viendo la evolución de la participación del grupo y el feedback recibido durante las sesiones y el último día de la intervención de promoción para la salud. En ese sentido, los objetivos establecidos para el proyecto de educación para la salud en la modalidad de ApS, fueron alcanzados satisfactoriamente.

Los participantes al finalizar cada una de las sesiones, demuestran haber entendido y adquirido la mayoría de los conocimientos impartidos a lo largo de las sesiones. Realizan preguntas y aportaciones enriquecedoras tanto para quienes conducían e impartían las sesiones (las alumnas de enfermería) como para el resto del grupo. Esto se vio evidenciado con la gran participación del grupo, incluso también para dar respuesta a las dudas de sus compañeros/as de grupo Por otro lado, los/las participantes también fueron capaces de realizar en el aula las actividades y ejercicios dinámicos explicados.

En la última sesión se realiza una puesta en común, en la que los/as participantes comparten como se han sentido y lo que han aprendido gracias a estas intervenciones. Así mismo, se muestran muy agradecidos y refieren que de cara a futuros programas estarían encantados de volver a participar.

La valoración del Trabajo Final de Grado en modalidad de ApS fue valorado muy positiva y satisfactoriamente por las alumnas que realizaron el proyecto. Mencionaron que el proyecto les llevó mucho tiempo y trabajo, pero que el aprendizaje obtenido y la experiencia vivida ha sido de un gran valor para ellas. Y utilizando sus propias palabras:

"Siento que he aprendido y me llevo mucho de este trabajo, pero no solo en cuanto a teoría o información sobre la Comuni- 
Promoción de la salud en personas sordas mayores de la comunidad sorda Meritxell Segovia-Quiñones • Vera Ponce-López, et al...

dad Sorda, sino a cómo interactuar con ella adaptándome a sus capacidades y teniendo en cuenta sus posibles limitaciones. Por lo que, en el caso de encontrarme, ya sea en el día a día, el hospital o cualquier servicio de salud, con alguna barrera de comunicación con una persona sorda, creo que gracias a este trabajo tendré herramientas para poder solucionar de forma efectiva esa situación." (Alumna participante del Proyecto)

“(...) ha sido un proyecto complejo debido a que es un proyecto muy completo. Pero, aun así, he sacado muchas cosas positivas de este trabajo y he tenido la oportunidad de conocer esta comunidad y de ofrecer mi ayuda para disminuir esta desigualdad en salud y tratar de que esta comunidad poco a poco disponga de todos los recursos que se merece." (Alumna participante del Proyecto)

\section{1}

\section{Consideraciones Finales}

Como aspectos a destacar en las consideraciones finales, es importante destacar la satisfacción tanto de las personas mayores de la Comunidad Sorda, de las alumnas y profesoras implicadas en el proyecto, pero además y como aspecto nuclear el impacto positivo en la vida y salud de estas personas participantes en la intervención. El resultado final de este trabajo es un claro ejemplo de lo que persiguen los proyectos de Aprendizaje y Servicio (ApS) en la Universitat de Barcelona, producir un aprendizaje riguroso y completo en el alumnado además de generar impacto positivo en la sociedad, en este caso en la Comunidad Sorda catalana. 
Promoción de la salud en personas sordas mayores de la comunidad sorda Meritxell Segovia-Quiñones • Vera Ponce-López, et al...

\section{Bibliografía}

ANGARita N. et. al. De que parlem quan diem envelliment actiu?. Barcelona: Fundació Salut i Envelliment UAB; 2017.

AZUA, P. Envejecimiento Activo. 2011. Disponible en: htTPs://www.cermi. ES/ES/ACTUALIDAD/NOVEDADES/EN-TORNO-AL-ENVEEECIMIENTO-ACTIVO. ACCESO EN: 20 Ост. 2020.

AZUA, P. Presentación. 2012. En: PéreZ L.; ANDReU, A. (Dir.) El ENVEJECIMIENTO dE LAS PERSONAS CON DISCAPACIDAD. DOCUMENTO DE POSICIÓN DEL Cermi Estatal. Madrid: Grupo Editorial Cinca, 2012. p. 11-13.

CABEZAS-PEÑA, C. et. al. Bases per a la promoció de L'envelliment actiu i saludable. Barcelona: Agència de Salut Pública de Catalunya; 2014. CDC. Centros para el control y la prevención de enfermedades: tipus de 22 PÉRDIDA AUDITIVA (SORDERA). DISPONIBLE EN: HTTPS://WWW.CDC.GOV/NCBDDD/ SPANISH/HEARINGLOSS/TYPES.HTML. ACCESO EN: 10 OctubRe 2019; 2019.

ChaVeiro, N. et. al. Atendimento à pessoa surda que utiliza a língua de sinals, na perspectiva do profissional da saúde. Cogitare Enfermagem, v. 15, N. 4, P. 639-645, 2010.

CHAVEIRO, N.; RODRÍGUEZ-MARTÍN, D.; FARIA, J. G.. EDUCAÇ̃̃o BILÍNGUE para surdos em Barcelona-Espanha. The Especialist, 2020, vol. 41, n. 1, 2020.

Departament de Salut. Resultats de l'enquesta de salut de Catalunya (ESCA). Generalttat de Catalunya, 2019 .Disponible en: http://Salutweb. GENCAT.CAT/CA/EL_DEPARTAMENT/ESTADISTIQUES_SANITARIES/ENQUESTES/ESCA/RESUlTATS_ENQUeSTA_SAlUt_CATALUNYA/. AcCeso en: 20 oct. 2020.

EBERHARD, D. M.; SIMONS, G. F.; FENNIG, C. D. Ethnologue: Languages of the World. American Sign Language. Twenty-second edition. Dallas: SIL InTERNATIONAL; 2019. 
Promoción de la salud en personas sordas mayores de la comunidad sorda Meritxell Segovia-Quiñones • Vera Ponce-López, et al...

GUTIERREZ, A.; VELASCO, C. La Respuesta educativa a los estudiantes con discapacidad auditiva, 2016. Edita la Organización de Estados IBEROAMERICANOS.

MORIN, V. et Al. AprendizAje-Servicio, COMPROMiso social e InNOVACIÓN. Experiencias en las asignaturas Enfermería Comunitaria y Trabajo Final de Grado. FeM: Revista de la Fundación Educación Médica, N. 20, P. S70-S72, 2017A.

MORIN et al. SeRVICE LeARning and heAlth education: InNovation IN NURSing education. Procedia-Social and Behavioral Sciences, n. 237, p. 956-961, 2017B.

MUÑOZ-BAELL, I. M et. AL. ComunidAdeS SORDAS: ¿PACIENTES O CIUDADANas?. Gaceta Sanitaria, v. 25, N. 1, P. 72-78, 2011.

OMS. Organización Mundial de la Salud. ¿Qué es la promoción de la SALUD?, 2016. DISPONIBLE EN: HTTPS://WWW.WHO.INT/FEATURES/QA/HEALTH-PROMOTION/ES/. ACCESO EN: 20 OCT. 2020.

OMS. Organización Mundial de la Salud. Sordera y pérdida de audición, 2019A. DISPONIBLE EN: HTTPS://WWW.WHO.INT/ES/NEWS-ROOM/FACT-SHEETS/DEIAIL/DEAFNESS-AND-HEARING-LOSS. ACCESO EN: 25 Oct. 2020.

OMS. Organización mundial de la salud: Salud y derechos humanos, 2019B. DISPONIBLE EN: HTTPS://WWw.WHO.INT/ES/NEWS-ROOM/FACT-SHEETS/DETAIL/HUMAN-RIGHTS-AND-HEALTH. ACCESO EN: 08 Oct. 2020.

OMS. Organización Mundial de la Salud. Envejecimiento y salud, 2019c. DISPONIBLE EN: HTTPS://WWW.WHO.INT/ES/NEWS-ROOM/FACT-SHEETS/DETAIL/ENVEIECIMIENTO-Y-SALUD. AcCeso en : 20 Oct. 2020.

PUiG, J. M. et. al. Aprendizaje servicio. Educar para la ciudadanía. BarceLona: Octaedro, 2009. 
Promoción de la salud en personas sordas mayores de la comunidad sorda Meritxell Segovia-Quiñones • Vera Ponce-López, et al...

RODRÍGUEZ-MARTíN, D. EL SILENCIO COMO METÁFORA. UNA APROXIMACIÓN A la Comunidad Sorda y a su sentimiento identitario. Perifèria. Revista dínvestigació I formació en Antropologia, Barcelona, v. 18, N. 1, P. 23-50, 2013. RODRÍGUEZ-MARTÍN, D.; RODRÍGUEZ, C.; FALCÓ-PEGUEROLES, A. ETHNOGRAPHIC ANALYSIS OF COMMUNICATION AND THE DEAF COMMUNITY'S RIGHTS IN THE clinical Context. Contemporay Nurse, London, v. 54, N. 2, P. 126-138, 2018. WFD. World Federation of Deaf. Who we are, 2019. Disponible en: HTTP://WFDEAF.ORG/WHO-WE-ARE/. ACCESO EN: 10 Sep. 2019.

\section{4}

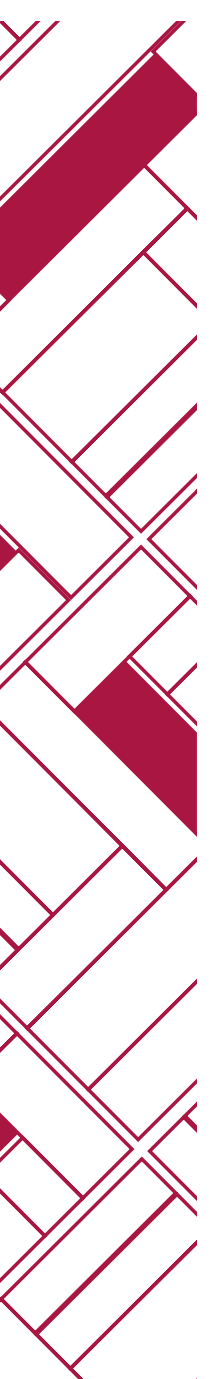

